\title{
ARTICLES \\ CONVERGENCE OF BAYES AND CREDIBILITY PREMIUMS
}

\author{
By Klaus D. SCHMIDT \\ University of Mannheim, West Germany
}

\begin{abstract}
For a risk whose annual claim amounts are conditionally i.i.d. with respect to a risk parameter, it is known that the Bayes and credibility premiums are asymptotically optimal in terms of losses. In the present note it is shown that the Bayes and credibility premiums actually converge to the individual premium.
\end{abstract}

\section{INTRODUCTION}

In this note we consider the classical model in experience rating:

Let $(\Omega, F, P)$ be a probability space, let $L^{2}(F)$ denote the Hilbert space of all random variables $\Omega \rightarrow \boldsymbol{R}$ having a finite second moment, and consider $\Theta, X_{1}, \ldots, X_{n}, X \in L^{2}(F)$. These random variables are interpreted as follows:

- $\Theta$ is a risk parameter which is observable or not and which determines the joint distribution of the annual claim amounts of the risk;

- $X_{1}, \ldots, X_{n}$ are the observable annual claim amounts of the risk over $n$ years in the past; and

- $X$ is the annual claim amount of the risk for a future year which is to be predicted by a premium $\delta^{*} \in \Delta$ minimizing the loss $E[X-\delta]^{2}$ over $\Delta$, where $\Delta \subseteq L^{2}(F)$ is a prescribed class of premiums to be specified below.

We assume that the following conditions are fulfilled:

- $X_{1}, \ldots, X_{n}, X$ are conditionally independent with respect to $\Theta$;

$-X_{1}, \ldots, X_{n}, X$ are conditionally identically distributed with respect to $\Theta$; and

$-\operatorname{var} E(X \mid \Theta)>0$.

Here $E(X \mid \Theta)$ denotes the conditional expectation of $X$ with respect to the $\sigma$-algebra $\sigma(\Theta)$ generated by $\Theta$, and we have $E(X \mid \Theta) \in L^{2}(\sigma(\Theta))$; correspondingly, $\operatorname{var}(X \mid \Theta)$ denotes the conditional variance $E\left((X-E(X \mid \Theta))^{2} \mid \Theta\right)$ of $X$ with respect to $\sigma(\Theta)$. Let $\bar{X}(n)$ denote the sample mean $\frac{1}{n} \sum_{i=1}^{n} X_{i}$, and define

$$
\begin{aligned}
\mu & :=E X=E[E(X \mid \Theta)] \\
\varphi & :=E \operatorname{var}(X \mid \Theta) \\
\lambda & :=\operatorname{var} E(X \mid \Theta)>0 \\
\kappa & :=\varphi / \lambda
\end{aligned}
$$


With these definitions, we have var $X=E[X-\mu]^{2}=\varphi+\lambda$; see also Lemma 2.1 below.

We consider four classes of premiums :

$$
\begin{aligned}
& \Delta_{0}:=\boldsymbol{R} \\
& \bar{\Delta}_{n}:=\operatorname{span}\left\{1, X_{1}, \ldots, X_{n}\right\} \\
& \Delta_{n}:=L^{2}\left(\sigma\left(X_{1}, \ldots, X_{n}\right)\right) \\
& \Delta_{\infty}:=L^{2}(\sigma(\Theta)) .
\end{aligned}
$$

Since each of $\Delta_{0}, \bar{\Delta}_{n}, \Delta_{n}, \Delta_{\infty}$ is a closed subspace of $L^{2}(\bar{F})$, the projection theorem in Hilbert spaces yields the existence of unique $\delta_{0}^{*} \in \Delta_{0}, \bar{\delta}_{n}^{*} \in \bar{\Delta}_{n}$, $\delta_{n}^{*} \in \Delta_{n}, \delta_{\infty}^{*} \in \Delta_{\infty}$ satisfying

$$
\begin{aligned}
& E\left[X-\delta_{0}^{*}\right]^{2}=\inf _{\Delta_{0}} E[X-\delta]^{2}, \\
& E\left[X-\bar{\delta}_{n}^{*}\right]^{2}=\inf _{\Delta_{n}} E[X-\delta]^{2}, \\
& E\left[X-\delta_{n}^{*}\right]^{2}=\inf _{\Delta_{n}} E[X-\delta]^{2}, \\
& E\left[X-\delta_{\infty}^{*}\right]^{2}=\inf _{\Delta_{\infty}} E[X-\delta]^{2} ;
\end{aligned}
$$

see BROCKWELL and DAVIS (1987; Theorem 2.3.1). In what follows we shall call

$$
\begin{array}{ll}
\delta_{0}^{*} & \text { the collective premium, } \\
\delta_{n}^{*} & \text { the credibility premium, } \\
\delta_{n}^{*} & \text { the Bayes premium, and } \\
\delta_{\infty}^{*} & \text { the individual premium. }
\end{array}
$$

We are mainly interested in the Bayes premium, which is the best prediction of $X$ by an arbitrary function of $X_{1}, \ldots, X_{n}$, and the credibility premium, which is the best prediction of $X$ by an affine-linear function of $X_{1}, \ldots, X_{n}$; see BAUER (1978; Lemma 55.1). The collective premium may be interpreted as the Bayes or credibility premium in the no-data case and serves mainly as a reference for comparisons. On the other hand, the individual premium should be expected to occur, in a sence to be made precise, as a limit of the Bayes and credibility premiums as the number of observables tends to infinity. It should, however, be noted that there is no obvious relation between $\Delta_{\infty}$ and $\Delta_{n}$ or $\bar{\Delta}_{n}$; this means that the subscript $\infty$ for the individual premium is, up to now, nothing more than a suggestive notation which still has to be justified.

In Section 2 of this note we recall some basic results concerning the identification of these premiums and of the losses attached to them. In Section 3 we discuss the asymptotic properties of the Bayes and credibility premiums.

\section{BASIC RESULTS}

Since $X$ and $X_{1}, \ldots, X_{n}$ are conditionally independent with respect to $\Theta$, the same is true for $X$ and each $\delta \in \Delta_{n}$. This yields the following useful result: 
2.1. Lemma. The identity

$$
E[X-\delta]^{2}=E[X-E(X \mid \Theta)]^{2}+E[E(X \mid \Theta)-\delta]^{2}
$$

holds for all $\delta \in \Delta_{n}$.

For the optimum premiums $\delta_{0}^{*}, \bar{\delta}_{n}^{*}, \delta_{n}^{*}, \delta_{\infty}^{*}$ we have:

\subsection{Proposition}

(a) $\delta_{0}^{*}=\mu$.

(b) $\bar{\delta}_{n}^{*}=\frac{\kappa}{\kappa+n} \mu+\frac{n}{\kappa+n} \bar{X}(n)$.

(c) $\delta_{n}^{*}=E\left(X \mid X_{1}, \ldots, X_{n}\right)=E\left(E(X \mid \Theta) \mid X_{1}, \ldots, X_{n}\right)$.

(d) $\delta_{\infty}^{*}=E(X \mid \Theta)$.

In particular, $\delta_{n}^{*}=E\left(\delta_{\infty}^{*} \mid X_{1}, \ldots, X_{n}\right)$.

For the losses attached to these premiums we have:

\subsection{Proposition}

(a) $E\left[X-\delta_{0}^{*}\right]^{2}=\varphi+\lambda$.

(b) $E\left[X-\bar{\delta}_{n}^{*}\right]^{2}=\varphi+\frac{\kappa}{\kappa+n} \lambda$

(c) $E\left[X-\delta_{n}^{*}\right]^{2}=\varphi+E\left[\operatorname{var}\left(E(X \mid \Theta) \mid X_{1}, \ldots, X_{n}\right)\right]$

(d) $E\left[X-\delta_{\infty}^{*}\right]^{2}=\varphi$.

In particular, $E\left[X-\delta_{\infty}^{*}\right]^{2} \leq E\left[X-\delta_{n}^{*}\right]^{2} \leq E\left[X-\bar{\delta}_{n}^{*}\right]^{2} \leq E\left[X-\delta_{0}^{*}\right]^{2}$.

In Propositions 2.2 and 2.3, assertion (a) is immediate, (d) follows from the fact that the projection of $X$ onto $\Delta_{\infty}=L^{2}(\sigma(\Theta))$ is precisely the conditional expectation of $X$ with respect to $\sigma(\Theta)$, and (c) follows from a similar argument combined with Lemma 2.1. Assertion (b) of Proposition 2.2 is due to BÜHLMANN $(1967,1970)$ and follows from the fact that the projection $\bar{\delta}_{n}^{*}$ of $X$ onto $\bar{\Delta}_{n}=\operatorname{span}\left\{1, X_{1}, \ldots, X_{n}\right\}$ satisfies $E\left[\left(X-\bar{\delta}_{n}^{*}\right) Z\right]=0$ for all $Z \in\left\{1, X_{1}, \ldots, X_{n}\right\}$, and hence $E X=E \bar{\delta}_{n}^{*}$ and $\operatorname{cov}\left(X, X_{j}\right)=\operatorname{cov}\left(\bar{\delta}_{n}^{*}, X_{j}\right)$ for all $j \in\{1, \ldots, n\}$. Assertion (b) of Proposition 2.3 is due to Jewell (1976) and is obtained by computing var $\left(X-\frac{n}{\kappa+n} \bar{X}(n)\right)$. The final inequality in Proposition 2.3 follows from $\varphi \leq \varphi+E\left[\operatorname{var}\left(E(X \mid \Theta) \mid X_{1}, \ldots, X_{n}\right)\right]$ and $\Delta_{0} \subseteq \bar{\Delta}_{n} \subseteq \Delta_{n}$. 


\section{ASYMPTOTIC CONSIDERATIONS}

As an immediate consequence of Proposition 2.3, the Bayes and credibility premiums are asymptotically optimal in terms of losses:

3.1. Lemma. $\lim E\left[X-\bar{\delta}_{n}^{*}\right]^{2}=\lim E\left[X-\delta_{n}^{*}\right]^{2}=E\left[X-\delta_{\propto}^{*}\right]^{2}$.

A slightly stronger result is the following:

3.2. Lemma. The Bayes premium $\delta_{n}^{*}$ and the credibility premium $\bar{\delta}_{n}^{*}$ converge in $L^{2}(\mathscr{F})$ to the individual premium $\delta_{\infty}^{*}$.

Proof. By Lemma 2.1 and Proposition 2.2, we have

$$
E\left[\delta_{\infty}^{*}-\delta\right]^{2}=E[X-\delta]^{2}-E\left[X-\delta_{\infty}^{*}\right]^{2}
$$

for all $\delta \in \Delta_{n}$, and the assertion now follows from Lemma 3.1.

Our main result is the following:

3.3. Theorem. The Bayes premium $\delta_{n}^{*}$ and the credibility premium $\bar{\delta}_{n}^{*}$ converge almost surely to the individual premium $\delta_{\infty}^{*}$.

Proof. By Proposition 2.2, we have

$$
\delta_{n}^{*}=E\left(X \mid X_{1}, \ldots, X_{n}\right),
$$

which means that the sequence $\left\{\delta_{n}^{*}\right\}$ is a martingale. Since $X \in L^{2}(7)$, there exists some $Z \in L^{2}(F)$ satisfying

$$
\lim \delta_{n}^{*}=Z \quad \text { a.s. and in } L^{2}(7) \text {; }
$$

see Neveu (1972; Proposition II.2.11). Since limits in $L^{2}(F)$ are unique, Lemma 3.2 yields

$$
Z=\delta_{\infty}^{*}
$$

and this implies $\lim \delta_{\mathrm{n}}^{*}=\delta_{\infty}^{*}$ a.s., which is the assertion for the Bayes premium. Furthermore, the conditional strong law of large numbers yields

$$
\lim \bar{X}(n)=E(X \mid \Theta) \quad \text { a.s., }
$$

and this implies $\lim \bar{\delta}_{\mathrm{n}}^{*}=\delta_{\infty}^{*}$ a.s., which is the assertion for the credibility premium.

The results of this section show that the Bayes and credibility premiums have the same desirable asymptotic properties. 


\section{REMARKS}

The importance of Hilbert space methods in experience rating, which is of course particular to the loss function considered here, was first pointed out by DEVylder (1976). A very nice introduction to Hilbert space theory with regard to applications in statistics may also be found in the monograph by Brockwell and DAvis (1987; Chapter 2).

The general form of Theorem 3.3 seems to be new, but special cases have been considered before: In the case where $\Theta$ is concentrated on the interval $(0,1)$ and the conditional distribution of $X$ is given by

$$
P(X=x \mid \Theta):=\left\{\begin{aligned}
1-\Theta, & \text { if } x=0 \\
\Theta, & \text { if } x=1,
\end{aligned}\right.
$$

which yields $E(X \mid \Theta)=\Theta$, the assertion concerning the Bayes premium occurs in the monograph by Billingsley $(1986 ;$ p. 496). In the case where $\Theta$ is discrete, the assertion concerning the credibility premium can also be obtained by partitioning $\Omega$ into the countable number of sets $\{\Theta=\theta\}$ with $P(\Theta=\theta) \neq 0$, renorming the probability measure on these sets, and applying the (unconditional) strong law of large numbers to the restrictions of $X_{1}, \ldots, X_{n}$ to the resulting new probability spaces; see Norberg (1979). In most models, however, $\Theta$ is assumed to be continuous, and in this case we have $P(\Theta=\theta)=0$ for all $\theta \in \boldsymbol{R}$ so that the previous argument fails.

It may be worthwhile to note that the martingale convergence theorem applied in the first part of the proof of Theorem 3.3 actually contains an identification of the limit, namely $Z=E\left(X \mid \mathscr{F}_{\infty}\right)$, where $\mathscr{F}_{\infty}$ denotes the $\sigma$-algebra generated by the infinite sequence $\left\{X_{n}\right\}$ of observables. In view of $\delta_{n}^{*}=E\left(\delta_{\infty}^{*} \mid X_{1}, \ldots, X_{n}\right)$, the same argument yields $Z=E\left(\delta_{\infty}^{*} \mid \mathscr{F}_{\infty}\right)$, and the point is that Lemma 3.2, which depends on the assumptions of the model, permits to conclude $Z=\delta_{\infty}^{*}$. The conditional strong law of large numbers applied in the second part of the proof of Theorem 3.3 is possibly known but difficult to find in the literature; for an elementary proof, see Schmidt (1990).

It is well-known that the Bayes and credibility premiums agree in many cases, but not always. Our final result contributes to the question, when these premiums agree:

4.1. Theorem. The following are equivalent :

(a) $\bar{\delta}_{n}^{*}=\delta_{n}^{*}$ for all $n \in N$.

(b) $\left\{\bar{\delta}_{n}^{*}\right\}$ is a martingale.

Proof. For all $n \in \mathbf{N}$, we have

$$
E\left(\bar{\delta}_{n+1}^{*} \mid X_{1}, \ldots, X_{n}\right)=\frac{\kappa+n}{\kappa+n+1} \bar{\delta}_{n}^{*}+\frac{1}{\kappa+n+1} \delta_{n}^{*},
$$


and hence $\bar{\delta}_{n}^{*}=\delta_{n}^{*}$ if and only if $E\left(\bar{\delta}_{n+1}^{*} \mid X_{1}, \ldots, X_{n}\right)=\bar{\delta}_{n}^{*}$.

This result is another example for the occurrence of martingales in connection with other distinguished properties of stochastic processes in insurance mathematics.

\section{ACKNOWLEDGMENT}

I would like to thank Professor J.O. Berger and the referees for their helpful comments on the first version of this paper.

\section{REFERENCES}

BAUER, H. (1978) Wahrscheinlichkeitstheorie und Grundzüge der Maßtheorie, 3. Auflage. DeGruyter, Berlin-New York.

Billingsley, P. (1986) Probability and Measure, Second Edition. Wiley, New York-Chichester. Brockwell, P.J. and Davis, R. A. (1987) Time Series: Theory and Methods. Springer, BerlinHeidelberg-New York.

BÜlmanN, H. (1967) Experience rating and credibility. ASTIN Bulletin 4, 199-207.

BUhlmanN, H. (1970) Mathematical Methods in Risk Theory. Springer, Berlin-Heidelberg-New York.

DeVylder, F. (1976) Geometrical credibility. Scandinavian Actuarial Journal, 121-149.

JEWELL, W.S. (1976) A survey of credibility theory. Operations Research Center Report No. 76-31. University of California, Department of Industrial Engineering and Operations Research, Berkeley. Neveu, J. (1972) Martingales à Temps Discret. Masson, Paris.

NorberG, R. (1979) The credibility approach to experience rating. Scandinavian Actuarial Journal, 181-221.

Sснмidt, K.D. (1990) Conditional laws of large numbers and applications. Preprint.

\section{KLAUS D. SCHMIDT}

Fakultät für Mathematik und Informatik, Universität Mannheim, A 5, 6800 Mannheim, West Germany. 